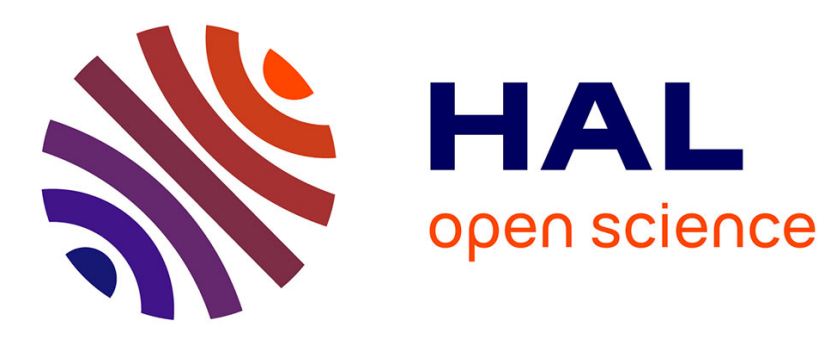

\title{
Substrate noise modeling with dedicated CAD framework for smart power ICs
}

Hao Zou, Yasser Moursy, Ramy Iskander, Camillo Stefanucci, Pietro Buccella, Maher Kayal, Jean-Michel Sallese

\section{- To cite this version:}

Hao Zou, Yasser Moursy, Ramy Iskander, Camillo Stefanucci, Pietro Buccella, et al.. Substrate noise modeling with dedicated CAD framework for smart power ICs. 2015 IEEE International Symposium on Circuits and Systems (ISCAS), May 2015, Lisbon, Portugal. pp.4, 10.1109/ISCAS.2015.7168943 . hal-01230110

\section{HAL Id: hal-01230110 https: / hal.sorbonne-universite.fr/hal-01230110}

Submitted on 17 Nov 2015

HAL is a multi-disciplinary open access archive for the deposit and dissemination of scientific research documents, whether they are published or not. The documents may come from teaching and research institutions in France or abroad, or from public or private research centers.
L'archive ouverte pluridisciplinaire HAL, est destinée au dépôt et à la diffusion de documents scientifiques de niveau recherche, publiés ou non, émanant des établissements d'enseignement et de recherche français ou étrangers, des laboratoires publics ou privés. 


\section{Substrate Noise Modeling with Dedicated CAD Framework for Smart Power ICs}

\author{
Hao Zou, Yasser Moursy, \\ and Ramy Iskander \\ Sorbonne Université, UPMC Univ Paris 06, \\ Paris, France \\ Email: hao.zou@lip6.fr
}

\author{
Camillo Stefanucci, Pietro Buccella, \\ Maher Kayal and Jean-Michel Sallese \\ Swiss Federal Institute of Technology (EPFL) \\ Lausanne, Switzerland CH-1015
}

\begin{abstract}
In smart power IC technology, low and high voltage circuits are integrated on the same substrate. The commutation of the high voltage circuits can induce substrate parasitic currents which can severely disturb the operation of the low voltage circuits. The parasitic currents due to minority carriers in the high voltage technology can be significantly high. However, the minority carrier propagation into the substrate is not considered in most of existing circuit simulators. In this paper, a novel computer-aided design tool for substrate parasitic extraction is proposed. A simple circuit with an injecting and a collecting $\mathrm{N}$ wells over a $P$-substrate is studied. With the distance between the wells varying, the lateral bipolar effect is illustrated. The spectre simulation results of extracted substrate equivalent circuit are compared to a TCAD simulation results. The comparison shows an acceptable relevant error. However, the simulation time was reduced by approximately 1400 times with respect to the TCAD.
\end{abstract}

\section{INTRODUCTION}

Integration of low voltage circuits with high voltage devices is used in smart power IC design [1]. However, unexpected substrate parasitic noise can severely disturb the sensitive nearby low voltage devices during the switching of power stage. Such substrate-noise coupling is considered the major cause of failures inside smart power integrated circuits [2]. The minority carriers propagation is still unpredictable and can cause failure after fabrication. This may increase the number of redesigns needed, increasing the cost and time-to-market [3].

Experimental extraction methodology of substrate-noise coupling was proposed in [4]. It is based on predicting a simple equivalent circuit from layout, or during conventional circuit simulation. However, it is not convenient for a complex circuit layout and lacks in accuracy. Today, none of the existing extraction methodologies consider minority carriers diffusion in the substrate of integrated circuit. Technology ComputerAided-Design (TCAD) [5] tools are the only tools available to interpret the impact of minority carriers. Despite physical based computer simulation are accurate, these are yet timeconsuming [6].

In this paper, we extend the substrate modeling approach initially proposed in [3], [7]. We show that this approach can be easily used to model lateral parasitic bipolar effects that accounts for minority and majority carriers propagation in the semiconductor substrate. An equivalent electrical schematic for the substrate is extracted by using a dedicated parasitic extraction tool. To illustrate the effectiveness of the approach, a layout with one injecting and one collecting $\mathrm{N}$-wells over a $\mathrm{P}$ substrate in studied. Results of circuit simulation is compared to a three dimensional TCAD simulation. The comparison shows that circuit simulation is very fast compared to TCAD simulations with an accuracy that is still quite acceptable.

The paper is organized as follows, in Section II a description of substrate parasitic models are illustrated. In Section III the architecture of extraction tool is presented. Comparison between circuit simulation of extracted substrate and TCAD simulation is discussed in Section IV. Finally conclusions are drawn in Section V.

\section{Modeling Methodology}

Fig. 1 shows a typical circuit configuration of Smart Power ICs. A $50 \mathrm{~V}$ power NDMOS is used as output transistor with a load connected to its drain. During the circuit switching this node goes below ground forward biasing the drain $\mathrm{N}$-well. Electrons are then injected in the substrate and are collected by nearby devices, as the isolation well of low-voltage NMOS. The resulting parasitic lateral NPN is difficult to characterize because its electrical characteristics are dependent on the distance $W_{b}$ between the two devices which is layout dependent. This distance corresponds to the substrate base of the parasitic BJT. To be able to simulate this coupling mechanism in standard design-flow an equivalent substrate network should be

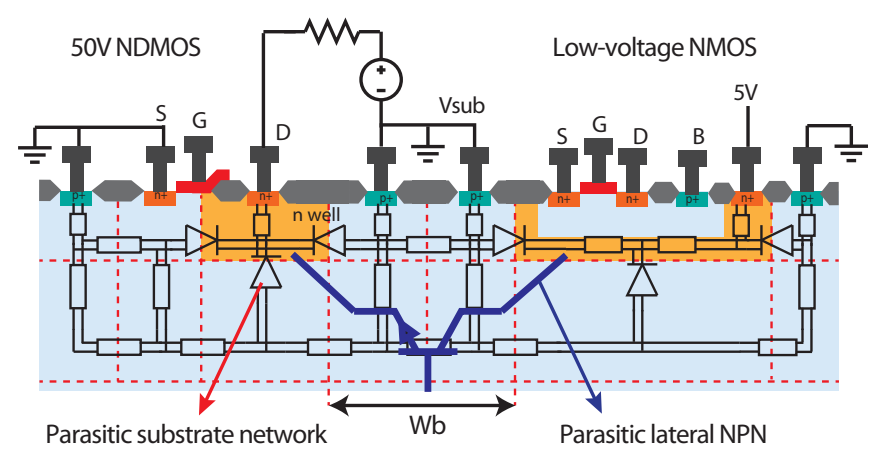

Fig. 1: Substrate equivalent circuit to model parasitic lateral NPN between high-voltage aggressor and low-voltage victim devices. 
extracted. This network cannot include compact model BJTs because their current gain is unknown and layout dependent. As proposed by [8], the extracted substrate circuit could be composed indeed of only diodes and resistances if minority carriers propagation is taken into account. The extraction of the substrate network should be done in an automatic way processing a general three-dimensional layout. The substrate must be divided in blocks (see red dashed lines in Fig. 1) and diodes or resistances should be properly instantiated. As a consequence, the parasitic lateral bipolar can be easily simulated if analyzing the layout we are able to detect PN junctions as injection or collector points of electrons, and to propagate the carriers through their interconnection by the means of diffusion resistances. The fundamental devices to consider for substrate currents modeling are then 4-terminal diodes and resistors able to carry physical voltages $V$ and currents $I$ but also minority carriers concentration (additional voltage $V_{e q}$ ) and gradient (additional current $I_{e q}$ ).

\section{CAD TOOL FOR SUBSTRATE SIMULATION}

The proposed methodology for substrate modeling relies on a 3D network of interconnected substrate parasitic components to represent the equivalent electrical substrate network. The principal idea is based on constructing a three dimensional meshing network, which is modeling the substrate by cuboid elements. Rectilinear layout-based meshing strategies are implemented to ensure minimum meshing for the extracted layout.

The diagram representing the substrate noise modeling flow using automatic extraction is depicted in Fig. 2. The entire CAD framework is developed by using "OpenAccess" [9] open source API. As reported in [6], the accuracy of substratenoise modeling is strongly dependent on the number of extracted parasitic components and its impact on the simulation time. At this first trial, we assume that minimal number of components is generated since the meshing is chosen to be minimal.

\section{A. Pre-processing stage}

Conventional extraction rules designed for substrate parasitic extraction have been introduced in [7]. In this work, a proposition of substrate extraction rules for the case studying is described as followings:

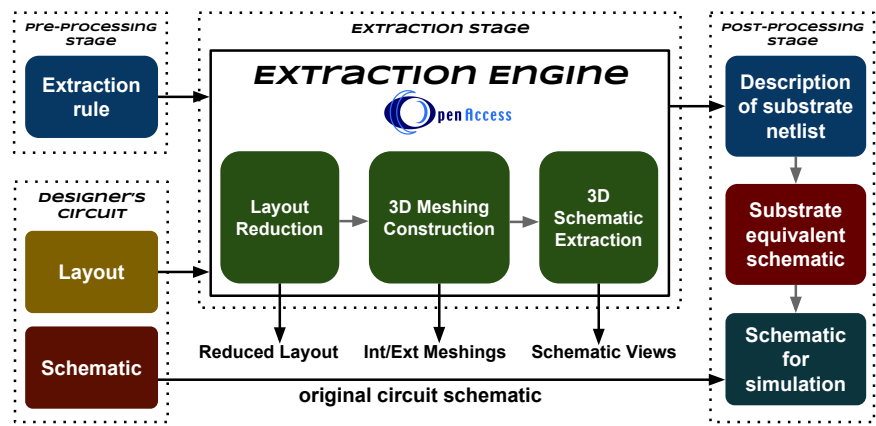

Fig. 2: The overall substrate parasitic extraction flow.

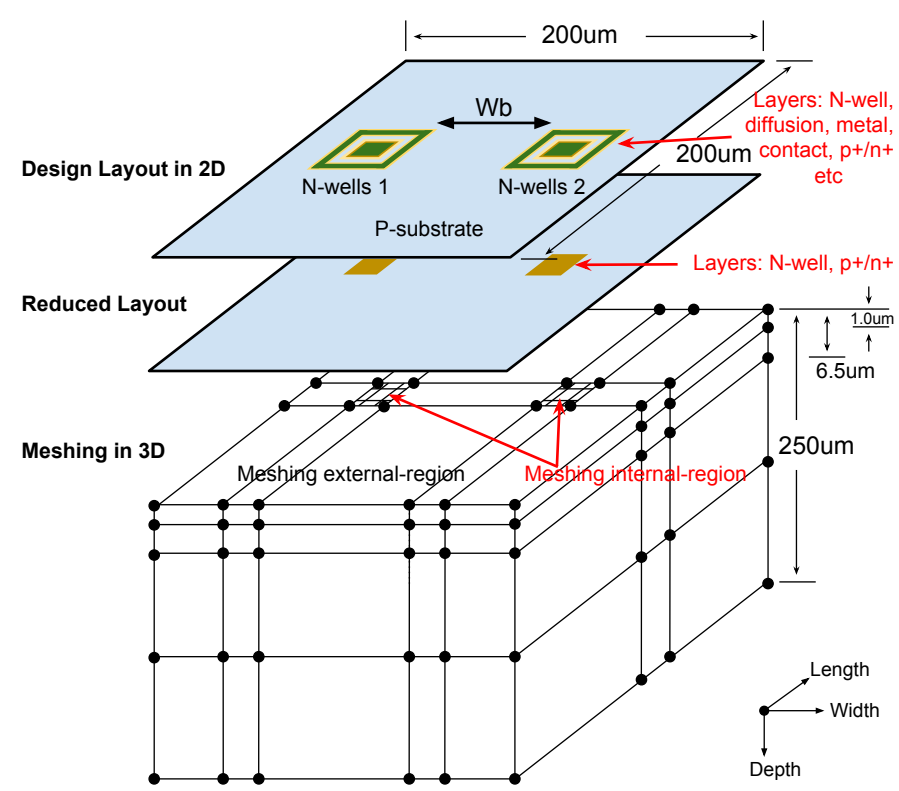

Fig. 3: Pre-processing of design layout.

- Layout reduction mechanism. Declare the layers not contributing to substrate parasitic, with "bypass" operation. It will actually neglect the indicated layers from layout database during extraction process and produce a reduced version of layout as presented in Fig. 3. Only $\mathrm{N}$-well layer is considered contributing to substrate parasitic in this simple case. Otherwise, $\mathrm{p}+\mathrm{n}+$ layer are related to homo-junction contact extraction [10].

- Definition of well-section. Consider the layout-dependent specifications given in Tab. I. Four well-sections are defined in "depth" direction (see mesh in 3D in Fig. 3). User-define extraction rules specifying the depth of each well-section are proposed in Tab. II, e.g. the depth of top well is $1.0 \mu \mathrm{m}$, considering the $\mathrm{p}+\mathrm{n}+$ homo-junction contact, which is actually the half of the depth to cuboid (from center to edge).

TABLE I: Layout-dependent specifications

\begin{tabular}{c|c|c|c}
\hline Layer & Length (for one) & Width (for one) & Depth \\
\hline $\mathrm{p}+$ (polygon) & $34.9 \mu \mathrm{m}$ & $34.9 \mu \mathrm{m}$ & $0.5 \mu \mathrm{m}$ \\
\hline $\mathrm{n}+$ & $20.1 \mu \mathrm{m}$ & $20.1 \mu \mathrm{m}$ & $0.5 \mu \mathrm{m}$ \\
\hline $\mathrm{N}-$ well & $20 \mu \mathrm{m}$ & $20 \mu \mathrm{m}$ & $6.5 \mu \mathrm{m}$ \\
\hline P-substrate & $200 \mu \mathrm{m}$ & $200 \mu \mathrm{m}$ & $250 \mu \mathrm{m}$ \\
\hline
\end{tabular}

TABLE II: User-define extraction rules

\begin{tabular}{c|c|c}
\hline Well-section & Depth & Layers \\
\hline top & $1.0 \mu \mathrm{m}$ & $\mathrm{p}+, \mathrm{n}+, \mathrm{N}$-well \\
\hline bottom1 & $5.5 \mu \mathrm{m}$ & $\mathrm{N}$-well \\
\hline bottom2 & $100.0 \mu \mathrm{m}$ & (P-substrate) \\
\hline bottom3 & $143.5 \mu \mathrm{m}$ & (P-substrate) \\
\hline
\end{tabular}




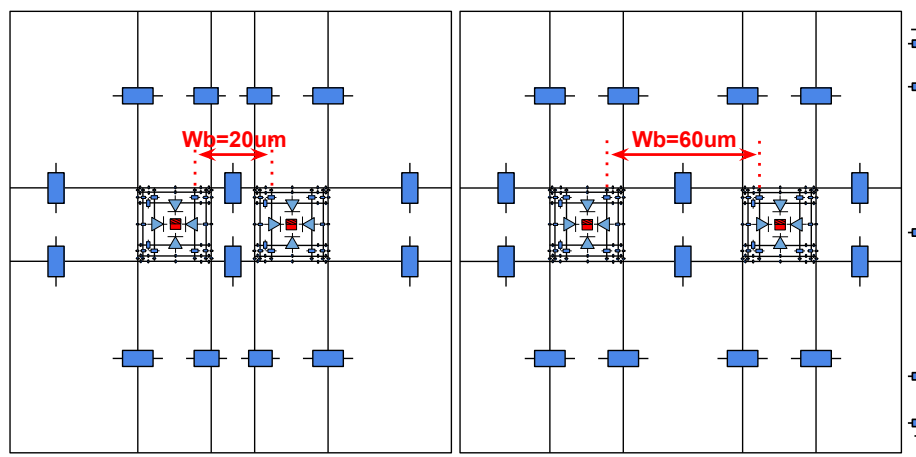

(a) top-layer for $W_{b}=20 \mu m$

(b) top-layer for $W_{b}=60 \mu m$

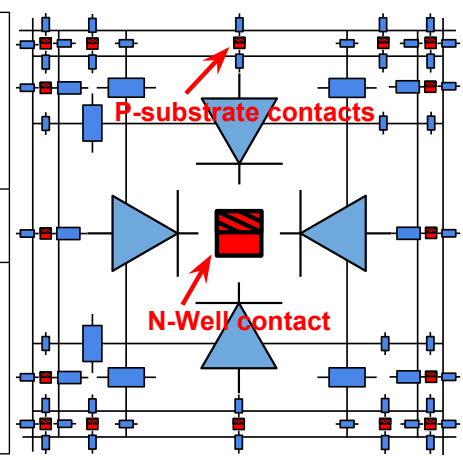

(c) focus on well region of (a)

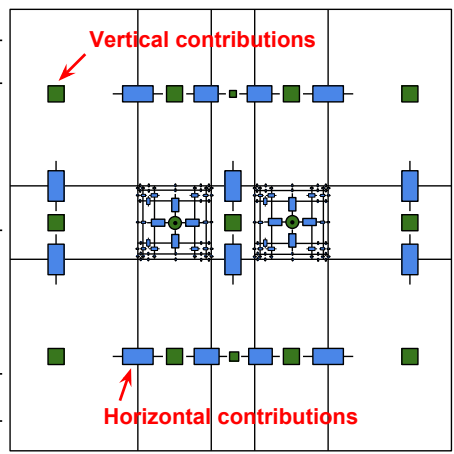

(d) bottom2-layer for $W_{b}=20 \mu m$

Fig. 4: Comparisons of meshing in 2D for $W_{b}=20 \mu \mathrm{m}$ (a) and $W_{b}=60 \mu \mathrm{m}$ (b) distance between the two wells, and extracted parasitic component for top-layer (a, b), N-well region (c) and bottom2-layer (d).

\section{B. Extraction stage}

At extraction stage as illustrated in Fig. 2, consider the OpenAccess layout database and conventional user-defined extraction rules as inputs. Then automatic extraction is performed on three steps as following:

- Layout reduction mechanism is performed at this moment and produce a reduced version of layout as illustrated in Fig. 3: $\mathrm{N}$-well layer and $\mathrm{p}+\mathrm{n}+$ layers are considered to have contributions to meshing network. This reduction mechanism saves significantly the computation time during extraction phase of large-scale integrated circuit.

- Meshing strategy relies on a three dimensional meshing strategy after scanning the reduced layout. Figures $4 \mathrm{a}$ and $4 \mathrm{~b}$ represent the meshing in 2D for different spacing between two N-wells (mesh rectangles in Fig. 4a for $W_{b}=20 \mu \mathrm{m}$ and Fig. $4 \mathrm{~b}$ for $W_{b}=60 \mu \mathrm{m}$ ).

- Component extraction mechanism is performed by going through the complete meshing in 3D and examining the neighbouring cuboids for horizontal and vertical contributions. Results of extraction are illustrated in Fig. 4. The blue resistance/diode components shown at Figures 4a, 4b represent the extraction horizontal for top layer. If we focus on the well region as Fig. 4c is presenting: four diodes are extracted surrounding $\mathrm{N}$-well which is over a P-substrate, homo-junction contacts are extracted where the " $p+$ " or " $n+$ ” present (see red rectangles). Vertical contributions are seen in bottom layer-sections which create the connection to the layer above. E.g. green rectangles in Fig. 4d represent the vertical resistances between bottom2-layer and bottom1-layer.

\section{Post-processing stage}

A CADENCE Design Systems [11] description language (SKILL) based program is embedded in CADENCE, and designated for components instantiation and placement of the complete substrate parasitics in the schematic environment during post-processing stage. The schematic can be simulated with a circuit simulator to see the effect of substrate noise.
For the purpose of further investigations, future developments will back-annotate the extracted substrate circuit to the original circuit.

\section{PARASITIC LATERAL NPN}

SPICE and TCAD simulations results were compared in order to validate the substrate model and the CAD extraction tool. Two three-dimensional structures (Fig. 5) including an injecting and a collecting $\mathrm{N}$-well over a P-substrate representing the parasitic NPN lateral transistor commonly encountered in Smart Power ICs were studied.

The common p-type substrate for both structures is a cube with dimensions $\mathrm{L}=200 \mu \mathrm{m}$ by $\mathrm{W}=200 \mu \mathrm{m}$ by $\mathrm{H}=250 \mu \mathrm{m}$. Both structures include two centred n-type wells placed at different distance: n-well spacing of $W_{b}=20 \mu \mathrm{m}$ and $W_{b}=60 \mu \mathrm{m}$ in the first and second structure. All n-type wells are the same with dimensions $\mathrm{L}=20 \mu \mathrm{m}$ by $\mathrm{W}=20 \mu \mathrm{m}$ by $\mathrm{H}=6.5 \mu \mathrm{m}$ and they are surrounded by a p+ doped ring $10 \mu \mathrm{m}$ wide. Each n-type well is connected out by a $\mathrm{n}+$ diffusion covering the entire well.

The P-substrate doping density profiles was assumed constant and equal to $\mathrm{Na}=4.5 \cdot 10^{15} \mathrm{~cm}^{-3}$. The n-wells doping density profiles were assumed to be Gaussian with peak doping density of $2 \cdot 10^{17} \mathrm{~cm}^{-3}$ and junction depth of $6.5 \mu \mathrm{m}$. In
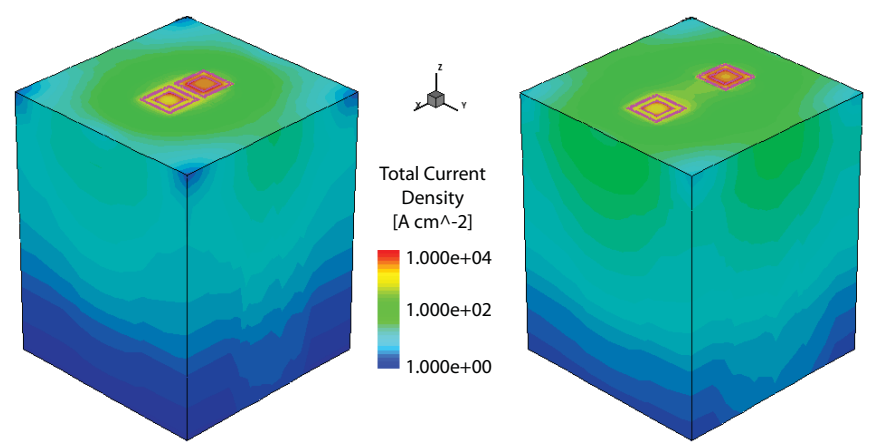

Fig. 5: Total current density from TCAD simulations at $V_{N 1}=$ $-1 \mathrm{~V}$ and $V_{N 2}=5 \mathrm{~V}$ for $W_{b}=20 \mu \mathrm{m}$ (left) and $W_{b}=60 \mu \mathrm{m}$ (right) distance between the two wells. 


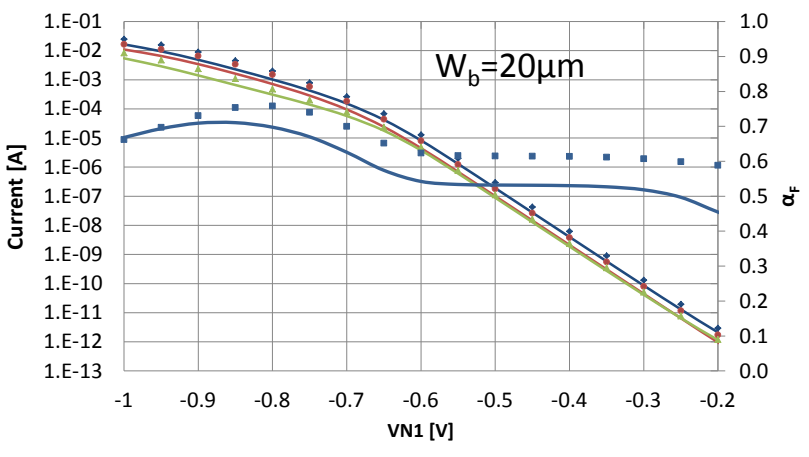

- IE_TCAD - IE_Spice - IC_TCAD —IC_Spice

IB_TCAD - IB_Spice $: \alpha F_{-} T C A D-\alpha F_{-}$Spice

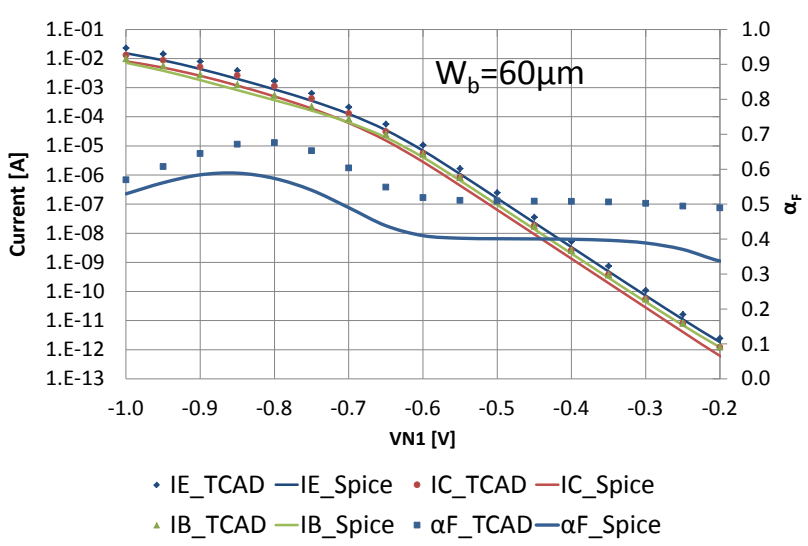

Fig. 6: comparison of simulated NPN Gummel plot with $V_{N 2}=$ $5 \mathrm{~V}$ for $W_{b}=20 \mu \mathrm{m}$ (left) and $W_{b}=60 \mu \mathrm{m}$ (right) distances.

the same way, doping density profiles for $\mathrm{p}+$ and $\mathrm{n}+$ doped diffusions were assumed to be Gaussian with peak doping density of $5 \cdot 10^{19} \mathrm{~cm}^{-3}$ and homojunction depth of $0.5 \mu \mathrm{m}$.

Using 3D Synopsys Sentaurus device simulator, the lateral NPN bipolar transistor gummel plot was derived. With the p-substrate biased at $0 \mathrm{~V}$ and the collector VN2 biased at $5 \mathrm{~V}$, the emitter voltage $\mathrm{VN} 1$ was swept from $0 \mathrm{~V}$ to $-1 \mathrm{~V}$ in order to activate the lateral NPN transistor. A lumped element resistance of $2 \Omega$ was tied to the emitter, base and collector terminal to mimic practical circuit metal connections.

The equivalent substrate netlists were extracted from both layouts to set up 3D SPICE simulations. Since the equivalent substrate model assumes abrupt $\mathrm{p}-\mathrm{n}$ junction, the doping

\begin{tabular}{|c|c|c|c|c|}
\cline { 3 - 5 } \multicolumn{2}{c|}{} & $\begin{array}{c}\text { Number of } \\
\text { Meshing } \\
\text { Elements }\end{array}$ & $\begin{array}{c}\text { Number of } \\
\text { DC Points }\end{array}$ & $\begin{array}{c}\text { Simulation } \\
\text { time [s] }\end{array}$ \\
\hline $\begin{array}{c}\text { Layout } \\
\text { Wb=20um }\end{array}$ & TCAD & 325000 & 21 & 2 hours \\
\cline { 2 - 5 } & SPICE & 510 & 101 & 5 sec. \\
\hline $\begin{array}{c}\text { Layout } \\
\text { Wb=60um }\end{array}$ & TCAD & 320000 & 21 & 2 hours \\
\cline { 2 - 5 } & SPICE & 510 & 101 & 5 sec. \\
\hline
\end{tabular}

TABLE III: TCAD and SPICE simulation time comparison concentration parameters used in the substrate electrical model are constant and equivalent to the averaged doping parameters derived from the device simulator. The simulation results are shown in Fig. 6, which shows the NPN Gummel plot comparison between TCAD and SPICE including the commonbase current gain parameter $\alpha_{F}$. Both simulations show how the parasitic lateral NPN currents and electrical parameter $\alpha_{F}$ depend on the distance $W_{b}$ between the two n-wells. Moreover SPICE simulations give accurate and reliable results on parasitic currents reducing simulation time considerably with respect to TCAD (See Table III).

\section{CONCLUSIONS}

Substrate modeling approach with dedicated extraction tool to model lateral parasitic bipolar effects has been presented. Extraction of electrical substrate network is done in seconds for two different wells spacing. Constructed meshing networks of substrate in $3 \mathrm{D}$ are illustrated in this paper. The lateral NPN transistor was simulated by SPICE and TCAD. In this example, the simulation time was reduced by approximately 1440 times with respect to the TCAD. The accuracy has been proved by comparison between device and SPICE simulation with an acceptable relevant error. We conclude that SPICE model has a strong advantage in the simulation time.

\section{ACKNOWLEDGEMENT}

This work is sponsored by the EU FP7 AUTOMICS project.

\section{REFERENCES}

[1] B. Murari, F. Bertotti, and G.Vignola, "Smart Power ICs," 2nd ed. Berlin, Germany: Springer-Verlag, 2002, pp. 218-220.

[2] Lo Conte, F.; Sallese, J.-M.; Kayal, M., "Modeling methodology of high-voltage substrate minority and majority carrier injections," SolidState Device Research Conference (ESSDERC), 2010 Proceedings of the European , vol., no., pp.194,197, 14-16 Sept. 2010.

[3] Y. Moursy et al., "AUTOMICS: A novel approach for substrate modeling", 18th IEEE European Test Symposium, May, 2013.

[4] Emoto, S.; Miyoshi, T.; Miyake, M.; Mattausch, H.J.; Miura-Mattausch, M.; Iizuka, T.; Sahara, Y.; Hoshida, T.; Matsuzawa, K.; Arakawa, T., "Experimental extraction of substrate-noise coupling between MOSFETs and its compact modeling for circuit simulation," Microelectronic Test Structures (ICMTS), 2012 IEEE International Conference on , vol., no., pp.101,104, 19-22 March 2012

[5] Synopsys Sentaurus Device, http://www.synopsys.com/tools/tcad

[6] Stefanucci, C.; Buccella, P.; Kayal, M.; Sallese, J.M., "Optimization strategy of numerical simulations applied to EPFL substrate model," Mixed Design of Integrated Circuits \& Systems (MIXDES), 2014 Proceedings of the 21st International Conference, vol., no., pp.334,337, 19-21 June 2014.

[7] Hao Zou; Moursy, Y.; Iskander, R.; Louerat, M.-M.; Chaput, J.-P., ”A novel CAD framework for substrate modeling" Microelectronics and Electronics (PRIME), 2014 10th Conference on Ph.D. Research in , vol., no., pp.1,4, June 30 2014-July 32014.

[8] F. Lo Conte, J. M. Sallese, M. Pastre, F. Krummenacher, and M. Kayal, "Global Modeling Strategy of Parasitic Coupled Currents Induced by Minority-Carrier Propagation in Semiconductor Substrates", IEEE Trans. Electron Devices, vol. 57, pp. 263-272, January 2010.

[9] OpenAccess programming interface for integrating design tools and design methodologies, www.si2.org.

[10] Stefanucci, C.; Buccella, P.; Kayal, M.; Sallese, J.M., "Impact of enhanced contact doping on minority carriers diffusion currents," Microelectronics and Electronics (PRIME), 2014 10th Conference on Ph.D. Research in , vol., no., pp.1,4, June 30 2014-July 32014.

[11] CADENCE: www.cadence.com 